\title{
Concerted Routing Metrics Evaluating and Distributed Rate Adaptation in Wireless Mesh Networks
}

\author{
${ }^{1}$ Sk.Farmanulla, ${ }^{2}$ P. Prasanna Murali Krishna, ${ }^{3}$ Dr.M.V.Subramanyam \\ ${ }^{I}$ M.Tech, Pursuing M.Tech of E.C.E, Dr.Samuel George Institute Of Engineering \& Technology, \\ Markapur,Prakasam (Dist), A.P, India \\ ${ }^{2}$ M.Tech,P.hd, Associate. Professor in Dept of E.C.E, Dr.SGIT,Markapur, Prakasam (Dist),A.P, India. \\ Principal, Santhiram Engineering College, Nandyala.
}

\begin{abstract}
This work proposes MARA, a joint method for automatic rate selection and route quality evaluation in Wireless Mesh Networks. This method targets at avoiding the problem of inaccurate link quality estimates, common to main existing proposals of multi-hop wireless routing metrics. In this proposal, the statistics collected by the routing protocol are used by the rate adaptation algorithm to compute the best rate for each wireless link. This coordinated decision aims at providing better routing and rate choices. Simulation results indicate that MARA leads to an overall improvement in network performance.
\end{abstract}

Key Words: Wireless Mesh Networks, Routing Metrics, Rate Adaptation Algorithms.

\section{Introduction}

For the past few years, Wireless Mesh Networks (WMN) have experienced a huge increase in popularity. Current mesh solutions typically employ devices based on the IEEE 802.11standard. These devices are capable of operating at multiple transmission rates, varying from $1 \mathrm{Mbps}$ to $54 \mathrm{Mbps}$. However ,selecting the most suitable rate is not trivial, since there is a trade-off between link capacity and transmission range.

Furthermore, WMN nodes must implement a dynamic route discovery mechanism. One of the core elements of this mechanism is the routing metric. Even though there are a number of metrics with very coherent formulations, they all face the obstacle of obtaining consistent and reliable information about the quality of wireless links [1].

This work proposes a new approach for solving these two important problems in Wireless Mesh Networks: automatic rate adaptation (ARA) and routing metric assignment (RMA).

The idea is to use a cooperative cross-layer method, called Metric Aware Rate Adaptation (MARA). With this method ,the rate adaptation algorithm (RAA) is able to use statistics provided by the routing metric in order to compute the most suitable data rate. Conversely, aware of the chosen rate, the metric may provide better estimates for link costs.

\section{Related Work}

MARA is a proposal for both RMA and ARA, and therefore there are two lines of related works. To the best of our knowledge, MARA is the first proposal of a joint solution for these two problems.

In terms of routing metrics, the very first proposal is the Hop Count, which considers the best path to be the one with the lowest number of hops. Other metrics, known as quality aware metrics, improve performance by dynamically evaluating Characteristics of the links. One of the most success full proposals is the Expected Transmission Count metric (ETX), which tries to estimate the number of layer-2 transmissions necessary to successfully send a packet between two nodes. The ETX metric works by periodically broadcasting control packets to infer a packet loss probability (PLP). The expected number of link-layer retransmissions is then the reciprocal of the PLP. Other variations of routing metrics were proposed based on ETX, such as the Expected Transmission Time metric(ETT), which tries to minimize the end-to-end delay, and the Minimum Loss metric (ML), which minimizes the end-to-end loss probability. For a more detailed explanation on these and other metrics, please refer to [1].

Although these three metrics try to model different network aspects, they are all based on the same PLP inference process.

This process is based on statistical data from periodical broadcast packets, which, according to the 802.11 standard, are always sent at a robust basic rate. However, since nodes usually use an RAA, data packets are typically sent at higher rates, in which packet loss rates are possibly higher. In other words, all these three metrics apply their models to potentially wrong statistics, leading to sub-optimal performance.

Some works based on the ETT metric suggest that link statistics should be based on uni cast probing packets for each neighbour. Despite generating more accurate statistics, this approach has serious scalability implications, especially on dense mesh networks. 
One of the simplest proposals of RAA is the Auto Rate Fallback algorithm (ARF). This algorithm maintains counters for sequences of success and failures of frame transmissions. If the failure counter passes a given threshold, the rate is decreased. There is also a threshold for the success counter, for which the rate is increased. The Sample Rate algorithm (SR) maintains a statistic about the mean frame transmission time (FTT) considering the rate, the mean number of retransmissions, and the 802.11 protocols overhead. When the algorithm detects that the mean FTT for the current rate is not the best, it changes the selection for the best one. The SNR algorithm, often considered to be optimal, chooses the highest rate possible such that the SNR on the receiver is sufficient to decode the frame.

III. The Mara Proposal

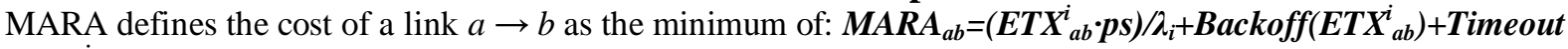
$\left(\boldsymbol{E T X}^{i}{ }_{a b}\right)$

where $\lambda i$ is the $i$-th available rate, $E T X_{a b}^{i}$ is the ETX oflink $a \rightarrow b$ at rate $\lambda_{i}, p s$ is the size of the probe packet, Backoff $\left(E T X_{a b}^{i}\right)$ is an estimate of the total back off time during the ETX ${ }_{a b}^{i}$ transmissions and TimeOut $\left(E T X_{a b}^{i}\right)$ is the total time spent by the sender waiting for the ack of lost frames. The cost of a path is the sum of costs of each link.

Clearly, MARA needs to calculate ETX at every possible rate. In order to do it, MARA estimates the mean SNR based on the broadcast rate, the estimated packet error rate, and probe packet size. Once the mean SNR is computed, this value can be used to estimate the packet error rate at other data rates. The computing of both SNR and packet error rate estimates is done using a pre-computed table that associates data rates ,packet sizes, SNR, and packet error rate. Given three of these values, the algorithm can compute an estimate for the fourth. function compute ETXAtRate $\left(P_{a b}, P_{b a}\right.$, Rate)

i

$S N R_{a b}=$ findSNRInTable $\left(P_{a b}\right.$,basicRate, probeSize);

$S N R_{b a}=$ findSNRInTable $\left(P_{b e}\right.$, basicRate,probeSize);

$N e w P_{a b}=1$-findPERInTable $\left(S N R_{a b}\right.$, RateprobeSize);

New $P_{b a}=1-$ findPERInTable $\left(S N R_{b a}\right.$,Rate,probeSize);

NewETX $=1 /\left(\mathrm{New} P_{a b} * \mathrm{New} P_{a b}\right)$;

return $($ NewETX);

\}

Although it is difficult to analytically build such table, previous works have already empirically done it [3]. Since SNR and packet error rate are not discrete greatnesses, it is impossible to build a table with all possible values. However, since the table resolution is high, it is possible to very accurately interpolate the two closest values.

Therefore, MARA is able to calculate good estimates for ETT at every available data rate, which makes routing decisions more precise. It is important to notice that this is achieved in a scalable manner using the same amount of overhead as the ETX metric. Moreover, since MARA does both metric and rate adaptation computing, the decisions are coordinated, avoiding mismatched choices.

\section{Simulation Results}

As a preliminary evaluation, MARA has been compared against 12 combinations of metrics (Hop Count, ETX, ETT, and ML) and RAAs (SR, SNR, and ARF). This comparison has been performed using the ns2 simulator. Since the standard 802.11 module of ns-2 does not implement multi-rate support, the 802.11 implementation found on [4] has been used. For this experiments, the implementation of MARA used tables available with the used 802.11 module [4]. For all metrics, the routing protocol was the Optimized Link State Routing protocol (OLSR) [1].

The topology used in the simulations is modeled after the real indoor wireless mesh network evaluated at [1]. Table I shows results based on a 300 seconds TCP flow between the two most distant nodes of the topology. The first column is the average throughput considering six executions with different seeds. The average and the $95 \%$ confidence interval clearly show that MARA outperformed all other combinations. On average, MARA was about 33\% better than the second best combination (ETX with SR). It is worth pointing out that MARA was able to outperform combinations using the SNR algorithm, which is nearly optimal. This result shows the importance of a synchronized decision between RMA and ARA. 


\section{TABLE I}

Throughput Results (In Kbps) For All The

13 EVALUATED COMBINATIONS.

\begin{tabular}{|c|c|c|}
\hline Mechanism & verage & Conf. Int. (95\%) \\
\hline MARA & 1108.37 & 15.35 \\
\hline ETX SR & 828.61 & 28.88 \\
\hline$M L S R$ & 521.90 & 27.49 \\
\hline$M L S N R$ & 425.65 & 4.15 \\
\hline$M L$ ARF & 382.46 & 3.77 \\
\hline ETT SNR & 374.40 & 7.70 \\
\hline ETX SNR & 354.30 & $1 . .67$ \\
\hline ETX ARF & 335.80 & 1.18 \\
\hline ETT SR & 335.35 & 18.97 \\
\hline ETT ARF & 288.63 & 6.32 \\
\hline HOP SNR & 67.79 & 5.24 \\
\hline HOP ARF & 34.79 & 3.72 \\
\hline HOP SR & 9.65 & 1.68 \\
\hline
\end{tabular}

\section{Conclusion}

This work proposes MARA, a cross-layer method for rateadaptation and route evaluation. Since MARA coordinatesboth rate selection and routing metric decisions, it guaranteesthat the selected data rate is always the optimal choice,considering the metric point of view. MARA also presentsa solution for the problem of inaccurate PLP estimates, byinferring the value of probabilities for every rate available.Another advantage of this proposal is its low overhead. MARA generates the same amount of control traffic, as the original ETX formulation.

Future work includes evaluating the possibility of making rate selection decisions for each packet, so that MARA could choose the best rate considering also the packet size. Since packet size directly influences the PLP, MARA could choose lower rates for bigger packets in un congested networks, for example. This idea be extended for routing decisions, so that different routes could be found for different sizes of packets.

\section{References}

[1] M. E. M. Campista, D. G. Passos, P. M. Esposito, I. M. Moraes,C. V. N. de Albuquerque, D. Muchaluat-Saade, M. G. Rubinstein, L. H.M. K. Costa, and O. C. M. B. Duarte, "Routing metrics and protocolsfor wireless mesh networks," IEEE Network, vol. 22, no. 1, pp. 6-12,Jan./Feb. 2008.

[2] K. Ramachandran, H. Kremo, M. Gruteser, P. Spasojevic, and I. Seskar,"Scalability analysis of rate adaptation techniques in congested IEEE802.11 networks: An orbit testbed comparative study," in WoWMoM2007, Jun. 2007, pp. 1-12.

[3] J. P. Pavon and S. Choi, "Link adaptation strategy for IEEE 802.11WLAN via received signal strength measurement," in ICC '03, vol. 2, 2003, pp. 1108-1113.

[4] C. E. Koksal and H. Balakrishnan, "Quality-aware routing metrics fortime-varying wireless mesh networks," IEEE Journal on Selected Areas in Communications, vol. 24, no. 11, pp. 1984-1994, Nov. 2006.

[5] R. Draves, J. Padhye, and B. Zill, "Routing in multi-radio, multi-hop wireless mesh networks," in ACM International Conference on MobileComputing and Networking (MobiCom), Sept. 2004, pp. 114-128.

[6] Special Interest research Group on NETworking (SIGNET). (2008, Jun.) dei80211mr: a new 802.11 implementation for ns 2. [Online]. Available: http://www.dei.unipd.it/wdyn/?IDsezione=5090 